\title{
The strong chromatic index of random graphs
}

\author{
Alan Frieze * $\quad$ Michael Krivelevich ${ }^{\dagger} \quad$ Benny Sudakov ${ }^{\ddagger}$
}

\begin{abstract}
The strong chromatic index of a graph $G$, denoted by $\chi_{s}(G)$, is the minimum number of colors needed to color its edges so that each colour class is an induced matching. In this paper we analyze the asymptotic behavior of this parameter in a random graph $G(n, p)$, for two regions of the edge probability $p=p(n)$. For the dense case, where $p$ is a constant, $0<p<1$, we prove that with high probability $\chi_{s}(G) \leq(1+o(1)) \frac{3}{4} \frac{n^{2} p}{\log _{b} n}$, where $b=1 /(1-p)$. This improves upon a result of Czygrinow and Nagle [2]. For the sparse case, where $n p<\frac{1}{100} \sqrt{\log n / \log \log n}$, we show that with high probability $\chi_{s}(G)=\Delta_{1}(G)$, where $\Delta_{1}(G)=\max \{d(u)+d(v)-1:(u, v) \in E(G)\}$. This improves a result of Palka [6].
\end{abstract}

\section{Introduction}

Given a graph $G=(V, E)$, the strong chromatic index $\chi_{s}(G)$ is the minimum number of colors needed to color the edges of $G$ so that every colour class is an induced matching i.e. any two edges of the same colour are at distance at least 2 in $G$. This notion was introduced by Erdős and Nešetřil (see [3]). Equivalently, it is the chromatic number of the square $L(G)^{2}$ of the line graph $L(G)$. Thus if $\Delta$ denotes the maximum degree of $G$, the maximum degree of $L(G)^{2}$ is at most $2 \Delta^{2}-2 \Delta$ and so $\chi_{s}(G) \leq 2 \Delta^{2}-2 \Delta+1$. It was conjectured in [3] that $\chi_{s}(G) \leq 5 \Delta^{2} / 4$ and this would be tight if true. Using a probabilistic argument Molloy and Reed [5] showed that $\chi_{s}(G) \leq(2-\varepsilon) \Delta^{2}$ for some small positive constant $\varepsilon$.

In this paper we study the strong chromatic index of the random graph $G(n, p)$. As usually, $G(n, p)$ stands for the probability space of labeled graphs on $n$ vertices, where every edge appears independently and with probability $p=p(n)$. Palka [6] showed that if $p=\Theta\left(n^{-1}\right)$ then $\mathbf{w h p}^{1} \chi_{s}(G)=O(\Delta(G))=$

*Department of Mathematical Sciences, Carnegie Mellon University, Pittsburgh PA15213, U.S.A. Supported in part by NSF grant CCR-0200945.

${ }^{\dagger}$ Department of Mathematics, Raymond and Beverly Sackler Faculty of Exact Sciences, Tel Aviv University, Tel Aviv 69978, Israel. E-mail: krivelev@post.tau.ac.il. Research supported in part by USA-Israel BSF Grant 2002-133, and by grant 64/01 from the Israel Science Foundation.

${ }^{\ddagger}$ Department of Mathematics, Princeton University, Princeton, NJ 08544, USA. Email address: bsudakov@math.princeton.edu. Research supported in part by NSF grants DMS-0355497, DMS-0106589, and by an Alfred P. Sloan fellowship.

${ }^{1} \mathrm{~A}$ sequence of events $\mathcal{E}_{n}$ occurs with high probability $(\mathbf{w h p})$ if $\lim _{n \rightarrow \infty} \operatorname{Pr}\left(\mathcal{E}_{n}\right)=1$ 
$O(\log n / \log \log n) . \mathrm{Vu}[7]$ showed that if $n^{-1}(\log n)^{1+\delta} \leq p \leq n^{-\varepsilon}$ for constants $0<\varepsilon, \delta<1$ then whp $\chi_{s}(G)=O\left(\Delta^{2} / \log \Delta\right)$. Czygrinow and Nagle [2] showed that if $p>n^{-\varepsilon}$ then $\chi_{s}(G) \leq(1+$ $o(1)) n^{2} p / \log _{b} n$ where $b=1 /(1-p)$. In this note we will obtain new bounds on $\chi_{s}(G(n, p))$ that improve the above results of Palka and of Czygrinow-Nagle.

To formulate our first theorem we need the following definition. For graph $G=(V, E)$ let $d(v)$ denote the degree of vertex $v \in V$ and let

$$
\Delta_{1}=\Delta_{1}(G)=\max \{d(u)+d(v)-1:(u, v) \in E\} .
$$

Set

$$
\lambda=\left(\frac{\log n}{\log \log n}\right)^{1 / 2},
$$

then for the sparse random graphs we prove the following tight result.

Theorem 1 Let $p$ be such that $n p \leq \lambda / 100$. Then whp, with $G=G(n, p)$,

$$
\chi_{s}(G)=\Delta_{1}(G)
$$

Remark 1 A straightforward calculation shows that in this range of edge probabilities, $\Delta_{1}(G)=$ $(1+o(1)) \Delta(G)$.

Remark 2 The observant reader will notice that our proof shows that the related choice number is also $\Delta_{1}$ whp i.e. as long as each edge is given a list of $\Delta_{1}$ colors, we can strongly edge color it.

We have learnt via private communication with Tomasz Łuczak that in unpublished work, he has obtained a result similar to Theorem 1.

For the dense case we improve the aforementioned result of Czygrinow and Nagle by a constant factor:

Theorem 2 Let $p>0$ be a constant. Denote $b=1 /(1-p)$. Then whp, with $G=G(n, p)$,

$$
\chi_{s}(G) \leq(1+o(1)) \frac{3}{4} \frac{n^{2} p}{\log _{b} n} .
$$

By the above result, the edges of $G(n, p)$ can be a.s. strongly colored so that the average size of a color class is at least $(1-o(1)) \frac{2}{3} \log _{b} n$.

Remark 3 The size of the largest induced matching in $G(n, p)$ is whp asymptotically equal to $\log _{b} n$ and so whp $\chi_{s}(G)$ is asymptotically at least $\frac{n^{2} p}{2 \log _{b} n}$. 


\subsection{Notation}

A sequence of events $\mathcal{E}_{n}$ is said to occur quite surely (qs), if $\operatorname{Pr}\left(\mathcal{E}_{n}\right)=O\left(n^{-K}\right)$ for any constant $K>0$. Unless the base is specifically mentioned, log will refer to natural logarithms.

We often refer to the Chernoff bound for the tails of the binomial distribution. By this we mean one of the following (see, e.g., [4]):

$$
\begin{aligned}
\operatorname{Pr}(B(n, p) \leq(1-\varepsilon) n p) & \leq e^{-\varepsilon^{2} n p / 2} \\
\operatorname{Pr}(B(n, p) \geq(1+\varepsilon) n p) & \leq e^{-\varepsilon^{2} n p / 3}, \quad \varepsilon \leq 1 \\
\operatorname{Pr}(B(n, p) \geq \mu n p) & \leq(e / \mu)^{\mu n p}
\end{aligned}
$$

\section{Sparse random graphs}

Given a graph $G$ with maximum degree $\Delta$, let $\beta=\sqrt{\Delta} / 2$. Denote by $L_{\beta}$ the set of vertices of $G$ which are within distance at most two from the set of vertices of degree at least $\beta$. Let $G_{\beta}$ be the subgraph of $G$ induced by $L_{\beta}$. First we need the following simple statement.

Lemma 3 Let $G$ be a graph for whose subgraph $G_{\beta}$ is acyclic. Then $\chi_{s}(G)=\Delta_{1}(G)$.

Proof. Clearly, for every edge $(u, v)$ of $G$, the edge itself and all edges incident with $u, v$ must have distinct colors. Therefore $\chi_{s}(G) \geq \Delta_{1}(G)$ and it remains to show the reverse inequality.

We start by coloring the edges of $G_{\beta}$. Since all connected components of this graph are trees it is enough to show that edges of every such tree $T$ can be colored using only $\Delta_{1}(T) \leq \Delta_{1}(G)$ colors. We do this by induction on the number of edges of $T$. It is trivial if $T$ has one edge or more generally is a star. Now root $T$ at an arbitrary vertex $r$ and let $x$ be a vertex of degree 1 of $T$ at maximum distance from $r$. Let $y \neq r$ be its unique neighbor in $T$ and let $z$ be the neighbor of $y$ on the path from $x$ to $r$ $\left(z=r\right.$ is possible here). Let $T^{\prime}=T-x$ and let $d, d^{\prime}$ refer to vertex degrees in $T, T^{\prime}$ respectively. By induction we can color the edges of $T^{\prime}$ using only $\Delta_{1}\left(T^{\prime}\right) \leq \Delta_{1}(T)$ colors. Then the number of colors forbidden for edge $e=(x, y)$ is at most

$$
D^{\prime}=d^{\prime}(y)+d^{\prime}(z)-1=(d(y)-1)+d(z)-1=(d(y)+d(z)-1)-1 \leq \Delta_{1}(T)-1 .
$$

Therefore there is a color which is not used at $y$ or $z$ and we can use it to color the edge $(x, y)$.

Having finished coloring the edges of $G_{\beta}$, we can color the remaining edges $e_{1}, e_{2}, \ldots, e_{M}$ of $G$ in this (arbitrary) order. Note that, by definition, for every edge $(u, v)$ outside $G_{\beta}$, all the neighbors of both $u$ and $v$ should have degree less than $\beta$. Therefore when we come to color $e_{i}$ we find that at most $2 \beta^{2} \leq \Delta / 2<\Delta_{1}$ colors have been forbidden by the coloring of previous edges and so there will always be an allowable color. 
Lemma 4 Let $p$ be such that $n p \leq \lambda / 100$. Let $T$ be a fixed set of vertices of size $|T|=t$ and let $A$ be a fixed set of at most $2 t$ edges. Then conditioning on the event that all edges in $A$ are present in $G(n, p)$, the probability that all the vertices in $T$ have degree at least $\lambda / 3$ is at most $2 e^{-\lambda t / 10}$.

Proof. By definition, it is easy to see that for such a set $T$, either there are at least $\lambda t / 9$ edges in the cut $(T, V(G)-T)$, or the set $T$ spans at least $\lambda t / 9$ edges of $G(n, p)$. Since we are conditioning on the presence of at most $2 t$ edges we have that either there are at least $\lambda t / 9-2 t \geq \lambda t / 10$ random edges in the cut $(T, V(G)-T)$, or similarly the set $T$ contains at least $\lambda t / 10$ random edges of $G(n, p)$. Using the fact that $n p \leq \lambda / 100$, the probability of the first event can be bounded by:

$$
\begin{aligned}
\left(\begin{array}{c}
t(n-t) \\
\lambda t / 10
\end{array}\right) p^{\lambda t / 10} & \leq\left(10 e(n-t) p \lambda^{-1}\right)^{\lambda t / 10} \\
& \leq e^{-\lambda t / 10}
\end{aligned}
$$

Similarly, the probability of the second event is at most

$$
\begin{aligned}
\left(\begin{array}{c}
t(t-1) / 2 \\
\lambda t / 10
\end{array}\right) p^{\lambda t / 10} & \leq\left(5 e(t-1) p \lambda^{-1}\right)^{\lambda t / 10} \\
& \leq e^{-\lambda t / 10}
\end{aligned}
$$

Altogether we obtain that the probability that all the vertices in $T$ have degree at least $\lambda / 3$ is at most $2 e^{-\lambda t / 10}$.

Lemma 5 Let $p$ be such that $n p \leq \lambda / 100$. Then whp, with $G=G(n, p)$, the subgraph $G_{\beta}$ is acyclic.

Proof. If $n p \leq 1 / \log \log n$ then the probability that $G(n, p)$ contains a cycle is at most $\sum_{t \geq 0} n^{t} p^{t}=$ $o(1)$, i.e., it is acyclic whp. Therefore we can assume that $n p \geq 1 / \log \log n$. In this case it is well known (see, e.g., [1]) that the maximum degree of the random graph is whp at least $(1+o(1)) \frac{\log n}{\log \log n}$. Let $X$ be the set of vertices of $G=G(n, p)$ which are within distance at most two from a vertex of degree at least $d_{0}=\lambda / 3$. Then it is enough to show that the subgraph of $G(n, p)$ induced by $X$ is acyclic whp.

Let $C$ be a shortest cycle in the subgraph $G[X]$ induced by $X$, and let $t$ be the length of $C$. We claim that there are at least $t / 10$ vertex disjoint paths of length at most 2 connecting vertices of the cycle to vertices of degree at least $d_{0}$. Since every vertex of the cycle is within distance at most two from some vertex of degree at least $d_{0}$, there is always at least one such path. Therefore we can assume that $t \geq 10$.

Let $v_{1}, \ldots, v_{s}$ be a largest set of vertices of $C$ such that the distance along the cycle between any two of them is at least 5 . Clearly $s=\lfloor t / 5\rfloor \geq t / 10$. Note that since $C$ was the shortest cycle in $G[X]$ the distance between every pair $v_{i} \neq v_{j}$ in this graph is also at least 5 . By the definition of $X$, for every $v_{i}, 1 \leq i \leq s$, there is a path $P_{i}$ of length at most two from $v_{i}$ to a vertex of degree at least $d$. 
All vertices of this path belong to $X$ and the paths $P_{i}$ and $P_{j}$ are vertex disjoint since otherwise the distance between $v_{i}$ and $v_{j}$ in $G[X]$ would be at most 4 . The path $P_{i}$ may share edges with $C$. On the other hand, once the path $P_{i}$ leaves the $C$ it cannot come back, since otherwise it will create a shorter cycle. Let $u_{i}$ be the last vertex of $P_{i}$ which still belongs to $C, P_{i}^{\prime}$ be the part of $P_{i}$ which is edge disjoint from $C$ and let $w_{i}$ be the endpoint of $P_{i}^{\prime}$ which have degree at least $d_{0}$. Denote by $H$ the union of all paths $P_{i}^{\prime}$ and $C$. We now estimate the probability that $G(n, p)$ contains such a subgraph. The number of ways to choose a cycle $C$ is at most $n^{t}$ and the probability that it appears in $G(n, p)$ is $p^{t}$. We can choose the set of vertices $u_{i}$ in at most $\left(\begin{array}{l}t \\ s\end{array}\right) \leq 2^{t}$ ways. The path between $u_{i}$ and $w_{i}$ can have length 0,1 or 2 and there are at most $3^{t / 5}$ different ways to choose a length for every path $P_{i}^{\prime}$. The number of paths of length $0,1,2$ is at most $1, n, n^{2}$ respectively and their existence probabilities are $1, p, p^{2}$. Note that after we choose the paths $P_{i}^{\prime}$ the vertices $w_{i}$ are fixed and we expose a set $A$ of at most $t+2(t / 5) \leq 2 t$ edges of $G(n, p)$. Therefore, by Lemma 4 , the probability that all the vertices $w_{i}$ have degree at least $d_{0}$ is bounded by $2 e^{-\lambda s / 10} \leq 2 e^{-\lambda t / 100}$. As $n p<\lambda<\sqrt{\log n}$, we can combine the above facts to conclude that the probability that a graph $H$ appears in $G(n, p)$ is bounded by

$$
\begin{aligned}
& \sum_{t \geq 3} n^{t} p^{t} 2^{t} 3^{t / 5}\left(1+n p+n^{2} p^{2}\right)^{t / 5} e^{-\lambda t / 100} \\
& <\sum_{t \geq 3}(6 n p)^{t}(2 \lambda)^{t / 5} e^{-\lambda t / 100} \\
& <\sum_{t \geq 3} \lambda^{2 t} e^{-\lambda t / 100} \\
& =o(1)
\end{aligned}
$$

This completes the proof of the lemma and the proof of Theorem 1.

\section{Dense random graphs}

Assume now that $0<p<1$ is a constant. We remind the reader that $b=1 /(1-p)$.

Let

$$
k=\left\lceil\left(\frac{2}{3}-\epsilon\right) \log _{b} n\right\rceil
$$

where $0<\epsilon<2 / 3$ is a constant. We will prove that whp $\chi_{s}(G(n, p)) \leq(1+o(1))|E(G)| / k$.

Let $s=\log ^{2} n, n_{0}=n / s$. Fix a partition of the vertex set $V(G)$ into $s$ parts $V_{1}, \ldots, V_{s}$ of nearly equal size: $\left|V_{i}\right| \approx n / s$. It will be enough to prove the following statement.

Lemma 6 With high probability $G=G(n, p)$ satisfies:

1. For all pairs $1 \leq i \neq j \leq s$, all but at most $O\left(n^{2} / \log ^{6} n\right)$ edges of the bipartite graph $G\left[V_{i}, V_{j}\right]$ can be packed into induced matchings of size $k$; 
2. For all pairs $1 \leq i \neq j \leq s,\left|E\left(V_{i}, V_{j}\right)\right| \leq n_{0}^{2} p+n_{0}^{3 / 4}$.

Indeed, assume that the conditions stated in the above lemma hold for $G$. Then we strongly color $E(G)$ as follows:

1. First, for each pair $1 \leq i \neq j \leq s$ color all but at most $n^{2} / \log ^{6} n$ edges between $V_{i}$ and $V_{j}$ in at most $\frac{\left|E\left(V_{i}, V_{j}\right)\right|}{k} \leq \frac{n_{0}^{2} p}{k}+\frac{n_{0}^{3 / 4}}{k}$ colors.

2. For each $1 \leq i \neq j \leq s$, color the uncolored edges between $V_{i}$ and $V_{j}$ in a new color each. The total number of additional colors used for all pairs $\left(V_{i}, V_{j}\right)$ does not exceed $\left(\begin{array}{c}s \\ 2\end{array}\right) \frac{n^{2}}{\log ^{6} n} \leq n^{2} / \log ^{2} n$.

3. Color all of the edges inside each $V_{i}$ in a new color. This stage consumes at most $s\left(\begin{array}{c}n / s \\ 2\end{array}\right) \leq$ $n^{2} / \log ^{2} n$ colors.

Altogether, we will have used $(1+o(1)) n^{2} p /(2 k)$ colors as required.

Proof of Lemma 6. Part (2) follows immediately from applying the Chernoff bounds for the binomial distribution to the number of edges joining $V_{i}, V_{j}$. We can thus concentrate on the bipartite graphs $G\left[V_{i}, V_{j}\right]$. Obviously, coloring such a bipartite graph is affected only by the edges between $V_{i}$ and $V_{j}$ and also the edges inside $V_{i}$ and $V_{j}$ (we are after the strong chromatic index here).

We first expose the edges of the random graph $G(n, p)$ inside the sets $V_{i}, 1 \leq i \leq s$. Let $t=n^{2 / 3}$. We will be able to assume that the following two properties hold inside each $V_{i}$ :

Lemma 7 With high probability in $G=G(n, p)$, for each set $V_{i}$ :

1. For every collection of $k$ disjoint sets $W_{1}, W_{2}, \ldots, W_{k} \subset V_{i}$ of size $\left|W_{i}\right|=\nu_{0} \geq n^{1 / 3} / \log ^{6} n$ there is an independent transversal in $G$, i.e., an independent set of vertices $\left\{w_{1}, w_{2}, \ldots, w_{k}\right\}$ such that $w_{i} \in W_{i}, i=1,2, \ldots, k$.

2. $V_{i}$ contains a collection $\mathcal{I}_{i}$ of $O\left(n^{5 / 3} / \log n\right)$ independent sets of size $k$ in $G$ such that $\left|I_{l_{1}} \cap I_{l_{2}}\right| \leq 1$ for each $I_{l_{1}} \neq I_{l_{2}} \in \mathcal{I}_{i}$, and each vertex $v \in V_{i}$ participates in $t(v)$ sets from $\mathcal{I}_{i}$, where $t(v) \in$ $\left[t \pm n^{5 / 9}\right]$.

Proof. For 1), fix $W_{1}, W_{2}, \ldots, W_{k} \subset V_{i}$ and let $X$ be the number of independent transversals. Then

$$
\mathbf{E}(X)=\nu_{0}^{k}(1-p)^{\left(\begin{array}{c}
k \\
2
\end{array}\right) .}
$$

We can now apply Janson's inequality, see for example Janson, Łuczak and Ruciński [4]. Thus let

$$
\begin{aligned}
\Delta & =\sum_{l=2}^{k}\left(\begin{array}{l}
k \\
l
\end{array}\right) \nu_{0}^{2 k-l}(1-p)^{2\left(\begin{array}{c}
k \\
2
\end{array}\right)-\left(\begin{array}{c}
l \\
2
\end{array}\right)} \\
& \leq k^{2} \nu_{0}^{2 k-2}(1-p)^{2\left(\begin{array}{c}
k \\
2
\end{array}\right)-1} .
\end{aligned}
$$


The last inequality follows form the fact that the sum is dominated by the term $l=2$. Indeed, the ratio of the $l$ th to the second term is

$$
\frac{\left(\begin{array}{l}
k \\
l
\end{array}\right) \nu_{0}^{2 k-l}(1-p)^{2\left(\begin{array}{c}
k \\
2
\end{array}\right)-\left(\begin{array}{l}
l \\
2
\end{array}\right)}}{\left(\begin{array}{l}
k \\
2
\end{array}\right) \nu_{0}^{2 k-2}(1-p)^{2\left(\begin{array}{c}
k \\
2
\end{array}\right)-1}} \leq \frac{k^{l-2}}{\nu_{0}^{l-2}(1-p)^{\left(\begin{array}{l}
l \\
2
\end{array}\right)-1}}=\left(\frac{k}{\nu_{0}(1-p)^{(l+1) / 2}}\right)^{l-2} \leq n^{-\epsilon(l-2) / 3} .
$$

Janson's inequality implies that

$$
\begin{aligned}
\operatorname{Pr}(X=0) & \leq \exp \left\{-\frac{\mathbf{E}(X)^{2}}{2 \Delta}\right\} \\
& \leq \exp \left\{-\frac{\nu_{0}^{2}(1-p)}{k^{2}}\right\}
\end{aligned}
$$

The number of choices for $i, W_{1}, W_{2}, \ldots, W_{k}$ is certainly less than $n^{k \nu_{0}}$ and so the probability there exists a collection without an independent transversal is at most

$$
n^{k \nu_{0}} \exp \left\{-\frac{\nu_{0}^{2}(1-p)}{k^{2}}\right\}=o(1) .
$$

To get 2), we can argue as follows. Observe that whp every set of $1 \leq j \leq k$ vertices of $V_{i}$ has $\left(1+O\left(n^{-1 / 6}\right)\right) n_{0}(1-p)^{j}$ common non-neighbors in $V_{i}$. Indeed, by the Chernoff bound the probability that there is a set $S,|S|=j \leq k$ for which the number of common non-neighbors lies outside $\left[(1 \pm \theta) n_{0}(1-p)^{j}\right]\left(\theta=n^{-1 / 6}\right)$ is at most

$$
2 \sum_{j=1}^{k}\left(\begin{array}{l}
n \\
j
\end{array}\right) \exp \left\{-\theta^{2} n_{0}(1-p)^{j} / 3\right\} \leq 2 \sum_{j=1}^{k}\left(\begin{array}{l}
n \\
j
\end{array}\right) e^{-n^{\varepsilon} /\left(3 \log ^{2} n\right)}=o(1) .
$$

This enables us to conclude that whp the number of independent sets $\tau(v)$ of size $k$ contained in

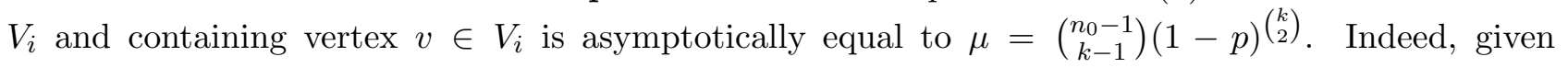
the above property, it follows by induction on $j \leq k$, that for all $v \in V_{i}$, there are between $(1-$ $\left.j n^{-1 / 6}\right)\left(\begin{array}{c}n_{0}-1 \\ j-1\end{array}\right)(1-p)^{\left(\begin{array}{c}j \\ 2\end{array}\right)}$ and $\left(1+j n^{-1 / 6}\right)\left(\begin{array}{c}n_{0}-1 \\ j-1\end{array}\right)(1-p)^{\left(\begin{array}{c}j \\ 2\end{array}\right)}$ independent sets of size $j$ in $V_{i}$ which contain $v$, i.e. $|\tau(v)-\mu| \leq n^{-1 / 6+o(1)} \mu$. Furthermore, we can also deduce that for a fixed pair $u, v \in V_{i}$ the number $\tau(u, v)$ of independent sets of size $k$ containing both $u$ and $v$ will be at most $(1+\theta)^{k}\left(\begin{array}{c}n_{0}-2 \\ k-2\end{array}\right)(1-p)^{\left(\begin{array}{c}k \\ 2\end{array}\right)-1}=$ $O\left(k \mu / n_{0}\right)$.

Form a random subfamily $\mathcal{I}_{i}^{0}$ of independent sets of size $k$ in $V_{i}$ by choosing each of them independently with probability $t / \mu$. Then, by the Chernoff bound, for every $v \in V_{i}$ qs the number of elements of $\mathcal{I}_{i}^{0}$ containing $v$ is between $\frac{\tau(v) t}{\mu}-t^{2 / 3}$ and $\frac{\tau(v) t}{\mu}+t^{2 / 3}$. Also,

$$
\left|\mathcal{I}_{i}^{0}\right|=\frac{\mu n_{0}}{k} \cdot \frac{t}{\mu}(1+o(1))=\frac{t n_{0}}{k}(1+o(1)) .
$$

For $u, v \in V_{i}$ the probability that $\mathcal{I}_{i}^{0}$ contains at least $\log n$ sets containing both $u$ and $v$ is at most

$$
\left(\begin{array}{c}
\tau(u, v) \\
\log n
\end{array}\right)\left(\frac{t}{\mu}\right)^{\log n} \leq\left(\frac{\tau(u, v) e t}{\mu \log n}\right)^{\log n}=\left(O\left(\frac{k t}{n_{0} \log n}\right)\right)^{\log n}=n^{(-1 / 3+o(1)) \log n},
$$


and thus qs for each pair $u, v \in V_{i}$

$$
\mathcal{I}_{i}^{0} \text { contains at most } \log n \text { sets containing } u \text { and } v \text {. }
$$

Also, observe that the number of pairs of independent sets of size $k$ in $V_{i}$, having $v$ and another vertex in common is at most $\sum_{u \in V_{i}}\left(\begin{array}{c}\tau(u, v) \\ 2\end{array}\right)=O\left(\mu^{2} k^{2} / n_{0}\right)$. Therefore, the probability that $\mathcal{I}_{i}^{0}$ contains at least $n^{1 / 2}$ disjoint pairs of independent sets containing $v$ and having another vertex in common is at most:

$$
\left(\begin{array}{c}
O\left(\mu^{2} k^{2} / n_{0}\right) \\
n^{1 / 2}
\end{array}\right)\left(\frac{t}{\mu}\right)^{2 n^{1 / 2}}=\left(O\left(\frac{\mu^{2} k^{2}}{n_{0}^{3 / 2}} \cdot \frac{t^{2}}{\mu^{2}}\right)\right)^{n^{1 / 2}}=n^{-(1 / 6-o(1)) n^{1 / 2}} .
$$

(Pairs $\left(I_{1}, I_{2}\right),\left(I_{3}, I_{4}\right)$ are disjoint if $\left\{I_{1}, I_{2}\right\} \cap\left\{I_{3}, I_{4}\right\}=\emptyset$.) We conclude that qs for all $v \in V_{i}$

$\mathcal{I}_{i}^{0}$ contains at most $n^{1 / 2}$ disjoint pairs of sets sharing $v$ and another vertex.

Assume now that properties (1) and (2) hold. Then we claim that for every $v \in V_{i}, \mathcal{I}_{i}^{0}$ contains at most $n^{1 / 2} \log ^{2} n$ pairs of independent sets sharing $v$ and another vertex. Suppose this is not so. Observe that by Property (1) each independent set $I \in \mathcal{I}_{i}^{0}$, containing $v$, has another vertex in common with at most $|I| \log n<\log ^{2} n-1$ sets from $\mathcal{I}_{i}^{0}$ containing $v$. Therefore, if we form a maximal by inclusion family of disjoint pairs in $\mathcal{I}_{i}^{0}$, containing $v$ and sharing another vertex, its size will be more than $n^{1 / 2} \log ^{2} n / \log ^{2} n=n^{1 / 2}-$ a contradiction to Property (2).

Deleting from $\mathcal{I}_{i}^{0}$ one independent set from each pair of sets sharing more than one vertex, we obtain a family $\mathcal{I}_{i}$ with $O\left(t n_{0} / k\right)=O\left(n^{5 / 3} / \log n\right)$ sets, in which each pair of sets has at most one vertex in common, and every $v \in V_{i}$ belongs to $t(v)$ sets from $\mathcal{I}_{i}$, where $t(v) \in\left[t-t / n^{1 / 7}, t+t / n^{1 / 7}\right]$.

From now on we assume that the conditions stated in Lemma 7 hold. Let us concentrate on the pair $\left(V_{i}, V_{j}\right), i<j$.

Let $v \in V_{i}$. Assume that $(u, v) \in E(G)$ where $u \in V_{j}$. We define

$$
R(v, u)=\left\{I_{l} \in \mathcal{I}_{i}: v \in I_{l}, N(u) \cap I_{l}=\{v\}\right\},
$$

i.e., $I_{l}$ is in $R(v, u)$ iff $v \in I_{l}$ and $v$ is the only neighbor of $u$ in $I_{l}$. Before diving into technical details, let us explain the main idea of the proof. Let $I_{l}=\left\{v_{1}, \ldots, v_{k}\right\}$. Assume that $u_{1}, \ldots, u_{k}$ form an independent set of size $k$ in $V_{j}$ such that $\left(v_{i}, u_{i}\right) \in E(G)$ and $I_{l} \in R\left(v_{i}, u_{i}\right)$ for all $1 \leq i \leq k$. Then the set of edges $\left\{\left(v_{i}, u_{i}\right): 1 \leq i \leq k\right\}$ forms an induced matching of size $k$ in $G$. Our aim will be to pack most of the edges between $V_{i}$ and $V_{j}$ in such matchings. To this end, we assign each edge $(v, u)$ of $G$ between $V_{i}$ and $V_{j}$ to one of the independent sets $I_{l} \in R(v, u)$. Then, for each set $I_{l} \in \mathcal{I}_{i}$ we distribute almost all the edges assigned to $I_{l}$ between induced matchings of size $k$ as indicated above.

Assume $e=(v, u) \in E(G)$ for $v \in V_{i}, u \in V_{j}$. We assign edge $e$ to one of the independent sets containing $v$ as follows: If $R(v, u)=\emptyset$ then $e$ stays unassigned, otherwise $e$ is assigned to a random member of $R(v, u)$. Denote

$$
\rho=(1-p)^{k-1}=n^{-2 / 3+\epsilon+o(1)} .
$$


Recall that we denoted by $t(v)$ the number of independent sets in $\mathcal{I}_{i}$ containing $v$. The probability that $e$ stays unassigned, conditioned on $e \in E(G)$, is $(1-\rho)^{t(v)} \leq e^{-n^{\epsilon+o(1)}}$. Therefore applying the union bound we can conclude that qs every edge $e=(u, v)$ of every pair $\left(V_{i}, V_{j}\right)$ gets assigned. Also,

$$
\begin{aligned}
\operatorname{Pr}\left[e \text { gets assigned to } I_{l}\right] & =\sum_{r=1}^{t(v)} \frac{1}{r}\left(\begin{array}{c}
t(v)-1 \\
r-1
\end{array}\right) \rho^{r}(1-\rho)^{t(v)-r} \\
& =\frac{1}{t(v)}\left(1-(1-\rho)^{t(v)}\right)
\end{aligned}
$$

(The parameter $r$ above counts the number of independent sets in $R(v, u)$.)

Let now $I_{l} \in \mathcal{I}_{i}, v \in I_{l}$. Denote by $T(v, l)$ the set of neighbors $u$ of $v$ in $V_{j}$ such that $(v, u)$ is assigned to $I_{l}$. Note that for any two edges $e=(v, u)$ and $e^{\prime}=\left(v, u^{\prime}\right)$ the events $e$, $e^{\prime}$ gets assigned to $I_{l}$ depend on disjoint sets of pairs of vertices in $G(n, p)$ and are mutually independent. Hence, conditioned on the degree $d\left(v, V_{j}\right)$, the random variable $|T(v, l)|$ is distributed binomially with parameters $d\left(v, V_{j}\right)$ and $\frac{1}{t(v)}\left(1-(1-\rho)^{t(v)}\right)$. The degree $d\left(v, V_{j}\right)$ in turn is also distributed binomially with parameters $n_{0}=\left|V_{j}\right|$ and $p$. So, applying the Chernoff bound twice, we can argue that whp

$$
|| T(v, l)\left|-n_{0} p / t\right| \leq n^{3 / 10}
$$

for all $\left(V_{i}, V_{j}\right), I_{l} \in \mathcal{I}_{i}, v \in I_{l}$.

Now consider $I_{l} \in \mathcal{I}_{i}$. Assume $I_{l}=\left\{v_{1}, \ldots, v_{k}\right\}$. The sets $T\left(v_{i}, l\right)$ are pairwise disjoint by construction. As long as $\left|T\left(v_{i}, l\right)\right| \geq n^{1 / 3} / \log ^{6} n$, we repeat the following procedure:

1. Find an independent transversal for the family $\left\{T\left(v_{i}, l\right)\right\}_{i=1}^{k}$. Let it be $\left(u_{1}, \ldots, u_{k}\right)$, where $u_{i} \in$ $T\left(v_{i}, l\right)$. This is possible due to the first condition of Lemma 7 ;

2. The set of edges $M=\left\{\left(v_{1}, u_{1}\right), \ldots,\left(v_{k}, u_{k}\right)\right\}$ forms an induced matching. We color $M$ by a fresh color;

3. Update $T\left(v_{i}, l\right):=T\left(v_{i}, l\right)-u_{i}$ for $1 \leq i \leq k$.

When this process stops, $\left|T\left(v_{i}, l\right)\right| \leq n^{1 / 3} / \log ^{6} n+2 n^{3 / 10}$, due to (3), and hence

$$
\left|\bigcup_{i=1}^{k} T\left(v_{i}, l\right)\right| \leq\left(\frac{n^{1 / 3}}{\log ^{6} n}+2 n^{3 / 10}\right) k=O\left(\frac{n^{1 / 3}}{\log ^{5} n}\right) .
$$

Since whp every edge is assigned to some $T(v, l)$, altogether, the number of edges between $V_{i}$ and $V_{j}$ that are left uncolored is at most

$$
\left|\mathcal{I}_{i}\right| \cdot O\left(\frac{n^{1 / 3}}{\log ^{5} n}\right)=O\left(\frac{n^{2}}{\log ^{6} n}\right) .
$$

This completes the proof of Lemma 6 and thus of Theorem 2 . 


\section{Concluding remarks}

- We strongly believe that the bound we obtain here for the dense case can be further improved, and in fact the following holds true: It was first conjectured in [2].

Conjecture 8 Let $p=p(n)$ satisfy $n^{-1+\epsilon} \leq p(n) \leq 0.99$, where $0<\epsilon<1$ is a constant. Then whp in the random graph $G=G(n, p)$,

$$
\chi_{s}(G)=(1+o(1)) \frac{n^{2} p}{2 \log _{b} n},
$$

where $b=1 /(1-p)$.

Proving this conjecture, even for a single value of $p(n)$, seems to be quite a challenging task. It appears that the proof method employed in the current paper has exhausted its potential, and new ideas are needed to establish the above conjecture.

- We prove that for random graph $G=G(n, p)$ where $n p \ll \sqrt{\log n / \log \log n}$ with high probability $\chi_{s}(G)=\Delta_{1}(G)$. A simple first moment calculation shows that this is no longer true when $n p \gg \sqrt{\log n}$. Hence the range of $p$ in the assertion of Theorem 1 is not very far from being best possible. Nevertheless, it would be interesting to determine or at least to estimate the edge probability threshold at which the equality $\chi_{s}(G)=\Delta_{1}(G)$ ceases to be valid.

\section{References}

[1] B. Bollobás, Random graphs, 2nd edition. Cambridge Studies in Advanced Mathematics 73, Cambridge University Press, Cambridge, 2001.

[2] A. Czygrinow and B. Nagle, Bounding the strong chromatic index of dense random graphs, Discrete Mathematics 281 (2004), 129-136.

[3] R. J. Faudree, A. Gyárfás, R. H. Schelp and Zs. Tuza, Induced matchings in bipartite graphs, Discrete Mathematics 18 (1989), 83-87.

[4] S. Janson, T. Łuczak and A. Ruciński, Random Graphs, John Wiley and Sons, New York, 2000.

[5] M. Molloy and B. Reed, A bound on the strong chromatic index of a graph, J. Combinatorial Theory Ser. B 69 (1997), 103-109.

[6] Z. Palka, The strong edge colorings of a sparse random graph, Australasian Journal of Combinatorics 18 (1998), 219-226.

[7] V. H. Vu, On some simple degree conditions which guarantee the upper bound on the chromatic (choice) number of random graphs, Combinatorics, Probability and Computing 11 (2002), 103-11. 